\title{
The Influence Machine: A Commentary on Hähnel and Martensen (2019)
}

\author{
DANIEL SHANAHAN[1] \\ Ohio State University
}

\begin{abstract}
This commentary discusses and contextualizes Hähnel and Martensen's analysis of Edison's recordings and correspondence, situating their study within some of the work done on the diffusion of innovations, and some other work on the history of recording. Their findings-that the mechanical limitations of recording possibly contributed to Edison's distaste for vocal vibrato-is mirrored in much of the work on early instrumental recordings, but whereas the effect for instrumental recordings was an increased vibrato, the solution for vocal recordings was the opposite.
\end{abstract}

Submitted 2019 September 4; accepted 2019 September 5.

KEYWORDS: style change, recording history, vibrato, Edison

RECORDING technology, like writing technology before it, at once disseminates and flattens the object. Like with the reverse question mark, at one point used to signify a rhetorical question (Watson, 2019), the interesting outliers often do not survive very long. In addition to the obvious points of the portability of music, or the shifting of musical performance from a cross-modal to a more auditory medium, there is this concretization of the musical object, often leading to a consolidation of musical styles and aspects of performance practice. Playing Raoul Koczalscki's brisk performance of Chopin's Op.28, no.4 will leave listeners scratching their heads, as clearly piano performances tend to become more homogenous over the course of the twentieth century. Some of this is possibly related to the broader aesthetic values that a culture holds regarding music. For example, Cook (2013) has argued that there is a shift from the interactive "read/write" culture that audiences had with music before recordings, to more of a "read only" culture which treats a piece of music as something to be consumed, rather than a roadmap open to some degree of interpretation. The influence of single performances on the broader practice becomes magnified in the age of recording, and is perhaps never more visible than when examining the history of vibrato.

Violinists had been using vibrato for hundreds of years, but it was considered a device to be used only occasionally (see Katz, 2004; p.85). Although it was mentioned in treatises, it was done so in the context of urging the performer to be prudent; in 1905, Joseph Joachim wrote that a player must "recognize the steady tone as the ruling one." (Brown, 1988; cited in Katz, p.86). The consistent use of vibrato seems to be a twentieth century phenomenon, and several reasons have been suggested as to why, ranging from the introduction of the chin rest in the $19^{\text {th }}$-century to the advent of metal strings after World War I.

Katz, however, argues that the recording technology itself had an outsized influence. The original recording devices were a challenge for violinists, and often picked up noises that would have been otherwise left unheard in a live performance (such as the sounds of bowing). Vibrato allowed for performers to play in a way that the pitch was more prominent than the other sounds, and in doing so performers were also able to hide issues with intonation, while nevertheless conveying to listeners the emotive aspects of a performance that would be lost in a purely auditory medium. Additionally, the vibrato itself was changed: what would work in a reverberant concert hall would not work in a recording studio, and so the speed of the vibrato increased. A likely candidate for the most influential performer to bring about this increase in usage is Franz Kreisler, whose early recordings did no less than change what the average listener thought a violin sounded like. In an age of millions upon millions of recordings available to stream at any given moment, it seems strange to think of a single individual having such an influence on an entire sound, resulting in a game of butterfly-effect-like speculation in which we might ponder what our musical landscape would look like if only very minor changes in performances had been made. If Kreisler, 
one of the most lauded violinists in history, can have such an effect on the sound of his instrument, it is quite interesting to note that the single individual who had the most impact on our perception of vocal performance is not a singer at all, but an (admittedly famous) inventor in New Jersey.

Hähnel and Martensen (2019) examine Edison's outsized role in bucking the trend of increasing vibrato, and combine evidence through Edison's correspondence as well as the analysis of recordings (both recordings meant for non-commercial purposes, and those that were already commercially available from other companies). Much like the example with the violin, treatises before the age of recording had preferred a steady tone (see the discussion of Sieber on p. 29). Hähnel and Martensen (2019) argue that Edison's perception of the nonmusical artefacts produced by the recording of vocal vibrato drove him to encourage its minimal use. Interestingly, this is the same reason for encouraging more vibrato in the violin; while "artefacts" are minimized by the vibrato in violin, they are highlighted by vibrato in the voice. Whereas "the pitch vibrato is likely to trigger artificial and audible volume changes" (p. 43) in the voice, those same volume changes might serve to mask the sounds of bowing while also creating a sense of a live performance, and the ambience and space that a recording would lack.

\section{SOME NON-MUSICAL ANALOGS}

We are left with the lasting influence of two individuals, each reifying the sound of an instrument through their influence despite being limited by the technology of the time. The findings are quite fascinating, and leave us with many questions on the nature of the transmission and diffusion of ideas, the limitations of such diffusion, and how technology can serve as both a motivator for change and a deterrent for future change. It might be useful to situate these findings within the larger framework of the diffusion of nonmusical innovations. Everett Rogers (2003) discusses how the Peruvian public health service attempted to introduce the practice of boiling water to remote villages. Very few families adopted the practice, primarily because it was perceived as "culturally inappropriate by the villagers." (p. 4); after all, one of the primary benefits of water was its cold temperature, and "[t]here are enough real threats in the world to worry about...without bothering about tiny animals that one cannot see, hear, touch, or smell." (p.4). Those who did adopt it were often social outsiders, and the practice therefore did not disseminate terribly well. It is interesting to note that, while in the case of the diffusion of violin vibrato, it was an "insider" who made the innovation-Kreisler was one of the most respected players of his day-whereas in the case of vocal vibrato (or lack thereof) it was simply a member with great social standing and the ability to control the technology.

Here, a second analogous point by Rogers might be the QWERTY keyboard. The original design of the keyboard was meant to slow down typists, as the mechanisms were likely to seize up in the event of too much action too quickly. As the mechanisms became more adept at handling fast typists, however, the QWERTY keyboard remained. When August Dvorak attempted to devise a more efficient keyboard layout, arranging the most commonly used letters on the same row, running experiments to gauge finger strength, and minimizing leaps as much as possible, it failed to be adopted. Rogers argues that, although technological innovations can be diffused quickly, the diffusion is often no match for a status quo, supported by vested interests (p.11). Hähnel and Martensen show a similar phenomenon: the proliferation of recordings in which preferences for sound were informed by mechanical limitations shaped a status quo that, to some extent, has stayed with us to this day.

\section{ACKNOWLEDGMENTS}

I'd like to thank David Aijón Bruno for pointing me to Mark Katz's Capturing Sound. This article was copyedited by Scott Bannister and layout edited by Diana Kayser.

\section{NOTES}

[1] Correspondence can be addressed to: Daniel Shanahan, Assistant Professor of Music Theory and Cognition, Ohio State University, 1899 College Road North, shanahan.37@osu.edu. 


\section{REFERENCES}

Brown, C. (1988). Bowing Styles, Vibrato and Portamento in Nineteenth-Century Violin Playing. Journal of the Royal Musical Association, 113(1), 97-128. https://doi.org/10.1093/jrma/113.1.97

Cook, N. (2013). Beyond the Score: Music as Performance. Oxford University Press. https://doi.org/10.1093/acprof:oso/9780199357406.001.0001

Hähnel, T., \& Martensen, K. (2019). How Thomas A. Edison shaped today's singing ideal: Tracking his ambiguous concept of tremolo by analysing archival documents and sound recordings. Empirical Musicology Review, 14(1-2), 22-49. https://doi.org/10.18061/emr.v14i1-2.6689

Katz, M. (2004). Capturing Sound: How Technology Has Changed Music. University of California Press.

Rogers, E. M. (2003). Diffusion of Innovations, 5th Edition. Simon and Schuster.

Watson, C. (2019). Semicolon: The Past, Present, and Future of a Misunderstood Mark. HarperCollins. 